\title{
MicroRNAs as critical regulators of the endothelial to mesenchymal transition in vascular biology
}

\author{
Jongmin $\mathrm{Kim}^{1,2,3, *}$ \\ ${ }^{1}$ Cellular Heterogeneity Research Center (CHRC), ${ }^{2}$ Research Institute of Women's Health (RIWH), ${ }^{3}$ Division of Biological Sciences, \\ Sookmyung Women's University, Seoul 04310, Korea
}

\begin{abstract}
The endothelial to mesenchymal transition (EndMT) is a newly recognized, fundamental biological process involved in development and tissue regeneration, as well as pathological processes such as the complications of diabetes, fibrosis and pulmonary arterial hypertension. The EndMT process is tightly controlled by diverse signaling networks, similar to the epithelial to mesenchymal transition. Accumulating evidence suggests that microRNAs (miRNAs) are key regulators of this network, with the capacity to target multiple messenger RNAs involved in the EndMT process as well as in the regulation of disease progression. Thus, it is highly important to understand the molecular basis of miRNA control of EndMT. This review highlights the current fund of knowledge regarding the known links between miRNAs and the EndMT process, with a focus on the mechanism that regulates associated signaling pathways and discusses the potential for the EndMT as a therapeutic target to treat many diseases. [BMB Reports 2018; 51(2): 65-72]
\end{abstract}

\section{INTRODUCTION}

Endothelial cells (ECs) line the inner surfaces of the blood vessels and lymphatic vessels in the body. ECs regulate vascular function by sensing and responding to various cues, and play a key role in the maintenance of vascular homeostasis (1). ECs have the capacity to undergo a dynamic cellular phenotypic switching, termed the endothelial to mesenchymal transition (EndMT), in response to local environmental cues throughout the vascular system. Since EndMT was initially described in relation to heart development (2), many studies have demonstrated the importance of the EndMT process during development (3-6). However, an

${ }^{*}$ Corresponding author. Tel: +82-2-710-9553; Fax: +82-2-2077-

7322; E-mail: jkim@sookmyung.ac.kr

https://doi.org/10.5483/BMBRep.2018.51.2.011

Received 30 December 2017

Keywords: Endothelial cell, Endothelial dysfunction, Endothelial to mesenchymal transition, MicroRNA, Vascular disease increasing number of studies have demonstrated that EndMT is closely associated with postnatal pathological processes including cancer progression (7), tissue fibrosis (8), pulmonary arterial hypertension (9), neointima formation (10, 11), vascular calcification (12) and atherosclerosis (13), as well as cerebral cavernous malformations (14). The EndMT features are similar to the extensively studied and better understood epithelial to mesenchymal transition (EMT). During EndMT, ECs lose their ability to express endothelial markers, such as vascular endothelial cadherin (VE-cadherin), platelet endothelial cell adhesion molecule (PECAM-1, also known as CD31) and von Willebrand Factor (vWF). Subsequently, ECs lose their endothelial characteristics and display mesenchymal phenotypes characterized by acquisition of a highly invasive and migratory potential and gain of expression of mesenchymal markers such as alpha smooth muscle actin ( $\alpha$-SMA), smooth muscle protein 22 alpha (SM22 $\alpha$ ), fibronectin, vimentin and fibroblast specific protein-1 (FSP-1) (Fig. 1) (7-15). Although the molecular mechanisms underlying EndMT are complex and still largely unclear, the EndMT has been gradually defined based on studies of EMT processes, which are better understood in terms of molecular and cellular mechanisms (16). The EndMT can be regulated by multiple extracellular cues, microRNAs (miRNAs), transcription factors and various signaling pathways in different tissues and various pathophysiological conditions. Among the many regulators that control the EndMT process, miRNAs are emerging as key regulators of the EndMT program. MiRNAs are a class of endogenous, small noncoding RNAs containing about 22 nucleotides that play an important role in post-transcriptional regulation of gene expression, typically through direct binding to the 3'-untranslated region of messenger RNA (mRNA) $(17,18)$. A single miRNA has the capacity to target multiple mRNAs; thus, it is not surprising that miRNAs affect the gene regulatory network and are involved in global cellular processes, including development, differentiation, cell death and cell proliferation (17-19). A growing number of studies have revealed that several miRNAs have the capacity to regulate EndMT processes and such regulatory roles of miRNAs in EndMT suggest potential therapeutic targets to prevent and treat many vascular diseases via modulation of miRNA levels. Here, this review highlights the emerging role of miRNAs during the EndMT process and 


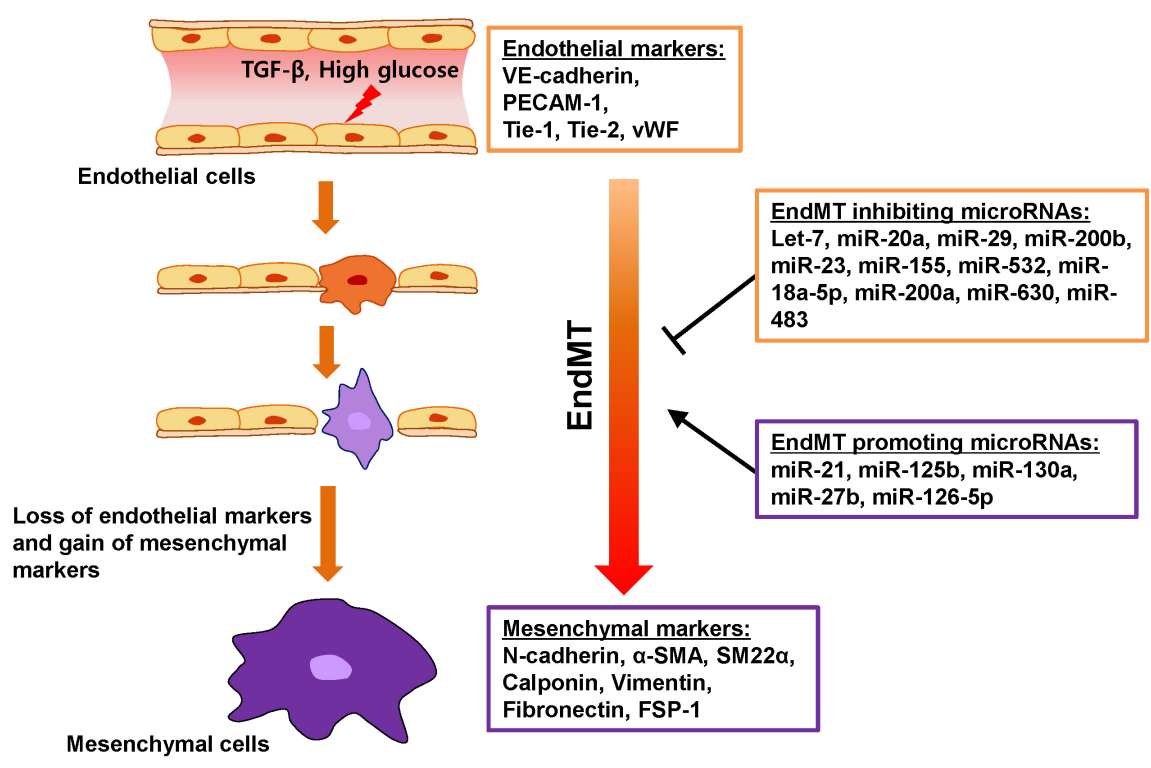

Fig. 1. MicroRNAs involved in EndMT. Schematic representation of significant miRNAs involved in EndMT regulation. During EndMT, ECs lose the expression of endothelial markers, such as VE-cadherin, PECAM-1, Tie-1, Tie-2, and vWF. Subsequently ECs gain of mesenchymal markers, such as $\mathrm{N}$-cadherin, $\alpha$-SMA, SM22 $\alpha$, fibronectin, vimentin and FSP-1. MiRNAs can promote or inhibit the EndMT program. Solid bars denote EndMT inhibiting miRNAs, solid arrows denote EndMT promoting miRNAs. discuss the potential for EndMT as a therapeutic target to treat vascular diseases.

\section{MICRORNA REGULATION OF THE ENDOTHELIAL TO MESENCHYMAL TRANSITION}

To date, a growing body of evidence shows that EndMT and EMT are primarily controlled by common signaling pathways, including transforming growth factor-beta (TGF- $\beta$ ) signaling, Notch signaling, and proinflammatory signaling cascades (20-22). These signaling pathways can activate or upregulate common transcription factors such as the Twist, Snail, Slug, zinc finger E-box-binding homeobox 1 (ZEB1) and ZEB2 (20-22). These transcription factors upregulate the expression of mesenchymal markers such as $\mathrm{N}$-cadherin, $\alpha$-SMA, SM22 $\alpha$, calponin, vimentin, fibronectin and FSP-1, although the precise molecular mechanisms are not fully understood. At the cellular level, these transcription factors can initiate transcriptional reprogramming and subsequently, ECs lose their apical-basal polarity and intercellular junctions, becoming mesenchymal-like cells during EndMT (20-23). While transcriptional control of EndMT has previously been studied extensively, an understanding of post-transcriptional control in this context has recently been sought, and investigated in several studies. Emerging studies have shown that miRNAs, key regulators of post-transcriptional regulation, are potent regulators of the EndMT process via targeting of key components associated with EndMT signaling pathways (Fig. 1) $(11,24)$.

\section{MICRORNAS THAT INHIBIT THE ENDOTHELIAL TO MESENCHYMAL TRANSITION}

\section{TGF- $\beta$ responsive miRNAs in EndMT}

Several miRNAs act to inhibit EndMT by directly targeting transcription factors or inhibiting signaling pathways associated with induction of EndMT. Among the signaling pathways that activate the EndMT process, the TGF- $\beta$ signaling represents the most well-known inducer of EndMT. Several studies have revealed that TGF- $\beta$ significantly downregulates the expression of several miRNAs (such as miR-200a, miR-20a, miR-29 and miR-630) leading to activation of the EndMT process (25-28). The miR-200 family is composed of five members; miR-200a, miR-200b, miR-200c, miR-141 and miR-429. Before the study of EndMT, it had been demonstrated that the miR-200 family had inhibitory effects on EMT through targeting of ZEB1 and ZEB2 (29). A recent study investigated the role of miR-200a (which is well known to inhibit the EMT process) as it relates to EndMT (25). It was demonstrated that miR-200a expression was significantly downregulated after treatment with TGF- $\beta 1$ of human aortic endothelial cells (HAECs), while TGF- $\beta 1$ treatment upregulated the expression of growth factor receptor-bound 2 (GRB2). Overexpression of miR-200a resulted in significant inhibition of EndMT via downregulation of FSP- 1 and $\alpha$-SMA and upregulation of VE-cadherin and PECAM-1. At the same time, miR-200a targets GRB2 mRNA, which plays a vital role in the modulation of fibrosis and regulates the expression of endothelial and mesenchymal markers including FSP-1, $\alpha$-SMA, VE-cadherin and PECAM-1 $(25,30,31)$. The expression of miR-20a, a member of the miR-17-92 cluster, is also regulated by TGF- $\beta 1$ treatment (26). In that study, Correia et al. demonstrated that the expression of 
miR-20a was decreased during TGF- $\beta$-induced EndMT, and miR-20a overexpression inhibited EndMT induction in human umbilical vein endothelial cells (HUVECs). During TGF- $\beta$ induced EndMT, endothelial marker VE-cadherin expression decreased while expression of mesenchymal marker SM22 $\alpha$ and mesenchymal transcription factors Snail1, Snail2 and Twist increased. These suppressive effects were partially reversed by overexpression of miR-20a. Interestingly, it was shown that transforming growth factor beta receptor I (TGFBR1, also known as ALK5), TGFBR2, and smad anchor for receptor activation (SARA, also known as ZFYVE9), which are key components of canonical TGF- $\beta$ signaling, were direct targets of miR-20a. Ectopic miR-20a expression decreased the levels of TGFBR1, TGFBR2 and SARA, and resulted in inhibition of EndMT (26). In addition to the miRNAs regulating key components of TGF- $\beta$ signaling during EndMT, there are miRNAs that target EndMT-inducing transcription factors activated by TGF- $\beta$, such as the Slug. The combination of TGF- $\beta$ and bone morphogenetic protein-4 (BMP-4) induces EndMT via downregulation of miR-630 expression in human dermal microvascular endothelial cells. The miR-630 was shown to directly target the Slug and inhibits EndMT (27). The MiR-29 is also downregulated in TGF- $\beta$-treated human dermal microvascular endothelial cells. Alteration of miR-29 expression is closely related to induction of EndMT via regulation of endothelial and mesenchymal markers and may be involved in the upregulation of dipeptidyl peptidase-4 (DPP-4) in kidneys of diabetic mice (28).

\section{MiRNAs in the Interaction between FGF and TGF- $\beta$ Signaling in EndMT}

Several studies have suggested that the fibroblast growth factor (FGF) signaling modulates TGF- $\beta$ signaling via regulation of many genes in various cell types (32-34). Fafeur et al. showed that treatment of FGF-2 in ECs inhibits the expression of the $85-k D a$ TGF- $\beta$ receptor subunit and attenuates the EC growth inhibitory activity of TGF- $\beta 1$ (32). It has also been found that FGF- 2 antagonizes TGF- $\beta 1$-mediated pericyte $\alpha$-SMA expression (33) and that TGF- $\beta$-induced EMT is reversed by FGF-1 through the MAPK/ERK kinase pathway (34). These studies indicate that the interaction between FGF and TGF- $\beta$ signaling may play a critical role in the regulation of the EndMT. Indeed, several studies have shown that endothelial FGF signaling counteracts TGF- $\beta$-induced EndMT via regulation of the expression of miRNAs including let-7 and miR-20a (11, 24, 26). Chen et al. demonstrated that disruption of baseline endothelial FGF signaling by knockdown of FRS2 (a key adaptor molecule involved in FGF signaling) decreased the level of let-7 miRNA which, in turn, increased expression of its target mRNA, TGF $\beta R$ 1. This served to produce activation of TGF- $\beta$ signaling, drove EndMT progression in human umbilical artery endothelial cells (HUAEC) and, in turn, led to neointima formation (11). Another study also showed evidence to substantiate the association of FGF signaling and let-7 in the induction of EndMT by the combination of TGF- $\beta 2$, IL- $1 \beta$ and TNF- $\alpha$, demonstrating that FGFR expression decreases in TGF- $\beta 2-$, IL- $1 \beta$ - and TNF- $\alpha$-treated HUVECs and leads to EndMT via downregulation of let-7 expression (24). In addition, it was also shown that FGF-2 increases miR-20a expression and regulates TGF- $\beta$ signaling in HUVECs by inhibiting TGFBR1, TGFBR2, and SARA expression via upregulated miR-20a, leading to inhibition of EndMT. These findings suggest that endothelial FGF signaling plays a critical role in maintenance of endothelial homeostasis by regulating TGF- $\beta$-induced-EndMT via modulation of miRNA expression (26). However, the correlation between FGF signaling and EndMT is still controversial. Several studies have found that IL-1 $\beta$, a key inducer of EndMT, upregulates the expression of FGF-2 through PI3K activation and, in turn, leads to EndMT of corneal ECs $(35,36)$, suggesting the role of FGF-2 as an inducer of EndMT in the context of inflammation caused by IL-1 $\beta$. This seeming controversy may be due to endothelial heterogeneity, i.e., ECs of different organs differentially respond to various stimuli (37). Thus, it is necessary to understand the molecular mechanism of EndMT in the context of endothelial heterogeneity.

\section{Other Important miRNAs in TGF- $\beta$-induced EndMT}

It has been reported that other miRNAs are also involved in TGF- $\beta$-induced EndMT. The MiR-23 negatively regulates TGF- $\beta$-induced EndMT in mouse embryonic endothelial cells (MEEC) and identified hyaluronic acid synthase 2 (Has2) as a direct target of miR-23. MiR-23 plays an essential role in cardiac valve formation by regulating Has2 expression (38). Bayoumi et al. showed that overexpression of miR-532 inhibits EndMT in cardiac ECs (CECs), while knockdown of the miR-532 display increased the EndMT (39). MiR-532 plays a key role in the regulation of cardiac vascularization via direct targeting of prss23 (which regulates Snail signaling to induce EndMT in HAECs), and knockdown of miR-532 inhibits CEC proliferation and cardiac vascularization after acute myocardial infarction (39). Moreover, endothelial miR-155 is upregulated by both TGF- $\beta$ stimulation and hypoxic conditions in MEECs, and the combination of TGF- $\beta$ stimulation and hypoxia was noted to be even more powerful in upregulation of miR-155 level and induction of the EndMT. Increased miR-155 downregulates RhoA expression, which is essential for the EndMT, and thereby functions as a negative feedback loop regulating EndMT (40). Overall, these studies may provide novel insights into the molecular mechanism(s) of miRNAs in TGF- $\beta$-driven activation of EndMT and in regulation of EndMT-mediated pathological phenotypes.

\section{MiRNAs Crosstalk during High Glucose-driven EndMT}

The molecular mechanisms of endothelial dysfunction by high glucose have been elucidated in the pathologic context of various vascular diseases (41). Emerging evidence has demonstrated that high glucose can lead to EndMT via 
regulation of the expression of miRNAs (such as miR-200b and miR-18a-5p), and contribute to the progression of diabetic complications (42-44). Cao et al. showed that high glucose led to decreased expression of miR-200b, concomitantly decreased expression of endothelial markers and increased expression of mesenchymal markers in human retinal microvascular endothelial cells. High glucose levels also upregulated the expression of TGF- $\beta 1$ and its downstream mediators of EndMT, such as Snail, Smad2 and p300, a known target of miR-200b. They further confirmed the role of miR-200b in the EndMT. EndMT was observed in the retinas of wild-type diabetic mice, but the process was prevented in miR-200b transgenic diabetic mice (42). This group also showed the role of miR-200b in EndMT in mouse heart endothelial cells in the context of diabetic cardiomyopathy (43). The MiR-18a-5p also inhibited EndMT via targeting Notch2 in the context of high glucose conditions in human aortic valvular endothelial cells. MiR-18a-5p expression was downregulated and, in turn, upregulated Notch2 expression after high glucose stimulation, resulting in the promotion of EndMT. In addition, ectopic miR-18a-5p expression decreased the levels of Notch2 (which is a target of miR-18a-5p), and subsequently inhibited the EndMT. The MiR-18a-5p overexpression also attenuated myocardial fibrosis in diabetic cardiomyopathy partially by inhibiting Notch2-mediated EndMT in vivo (44). Most of studies on the molecular mechanisms of EndMT have focused mainly on the TGF- $\beta$-mediated signaling pathway. However, further studies are also needed to understand the molecular mechanisms associated with EndMT induced by high glucose levels, inflammation, chemokines, growth factors and other stimuli.

\section{MICRORNAS THAT PROMOTE THE ENDOTHELIAL TO MESENCHYMAL TRANSITION}

On the contrary, several miRNAs have the capacity to promote EndMT by directly targeting molecules associated with inhibition of EndMT (45-49). For example, miR-21 expression increased during TGF- $\beta$-induced EndMT in HUVECs and inhibition of miR-21 has been shown to partially prevent TGF- $\beta$-induced-EndMT. In addition, it was found that miR-21 negatively regulated phosphatase and tensin homolog (PTEN), a well-known target of miR-21, and following activation of the Akt pathway, resulted in promotion of the EndMT. In vivo, miR-21 was upregulated in cardiac ECs during pressure overload-induced cardiac fibrosis, and it was attenuated by inhibition of miR-21 (45). It was reported that the level(s) of several miRNAs were differentially regulated during TGF- $\beta$ -

Table 1. MicroRNAs and target genes regulating the EndMT

\begin{tabular}{|c|c|c|c|c|c|}
\hline Endothelial cell type & microRNA & Target & Effect on EndMT & Clinical relevance & Reference \\
\hline HUAEC, HUVEC & Let-7 & TGF $\beta R 1$ & Inhibit & Neointima formation and fibrosis & 11,24 \\
\hline HAEC & miR-200a & GRB2 & Inhibit & Cardiac fibrosis & 25 \\
\hline HUVEC & miR-20a & TGF $\beta R 1$, TGF $\beta R 2$, SARA & Inhibit & Non determined & 26 \\
\hline HD-MVEC & $\operatorname{miR}-630$ & Slug & Inhibit & Heterotopic ossification & 27 \\
\hline HMVEC & miR-29 & DPP-4 & Inhibit & Diabetic nephropathy & 28 \\
\hline MEEC & miR-23 & Has2 & Inhibit & Cardiac valve formation & 38 \\
\hline CEC & miR-532 & PRSS23 & Inhibit & Acute myocardial infarction & 39 \\
\hline MEEC & miR-155 & RhoA & Inhibit & Non determined & 40 \\
\hline HRMEC & miR-200b & Smad2, Snail & Inhibit & Diabetic retinopathy & 42 \\
\hline MHEC & miR-200b & p300 & Inhibit & Diabetic cardiomyopathy & 43 \\
\hline HAVEC & miR-18a-5p & Notch2 & Inhibit & Diabetic cardiomyopathy & 44 \\
\hline HUVEC & miR-21 & PTEN & Promote & Cardiac fibrosis & 45 \\
\hline MCEC & miR-125b & p53 & Promote & Cardiac fibrosis & 46 \\
\hline MS-1 & $\operatorname{miR}-27 b$ & $\begin{array}{l}\text { Elk1, Neuropilin 2, Plexin A2, } \\
\text { Plexin D1 }\end{array}$ & Promote & Non determined & 47 \\
\hline LMVEC & miR-130a & BMPR2 & Promote & Pulmonary arterial hypertension & 48 \\
\hline RPMEC & $\operatorname{miR}-126-5 p$ & Non determined & Promote & Neonatal pulmonary hypertension & 49 \\
\hline HUVEC & miR-483 & CTGF & Inhibit & Kawasaki disease & 53 \\
\hline
\end{tabular}

HUAEC: human umbilical artery endothelial cell, HUVEC: human umbilical vein endothelial cell, HAEC: human aortic endothelial cell, HD-MVEC: human dermal microvascular endothelial cell, HMVEC: human dermal microvascular endothelial cells, MEEC: mouse embryonic endothelial cell, CEC: cardiac endothelial cell, HRMEC: human retinal microvascular endothelial cell, MHEC: mouse heart endothelial cell, HAVEC: human aortic valvular endothelial cell, MCEC: mouse cardiac endothelial cell, MS-1: mouse pancreatic microvascular endothelial cell, LMVEC: lung microvascular endothelial cell, RPMEC: rat pulmonary microvascular endothelial cell. 
induced EndMT in mouse cardiac endothelial cells (MCECs) (46). Measured by miRNA array analysis, it was found that miR-125b, let-7c, let-7g, miR-21, miR-30b and miR-195 were upregulated and miR-122a, miR-127, miR-196 and miR-375 were downregulated during TGF- $\beta$-induced EndMT. Among these miRNAs, upregulation of miR-125b expression was validated in TGF- $\beta$-induced EndMT-derived cells and control MCECs by miRNA qPCR analysis. The expression of p53, a target of miR-125b and antagonizing TGF- $\beta$-induced profibrotic responses was significantly inhibited during EndMT of MCECs, which suggested the possibility that increased miR-125b downregulates p53, thereby promoting EndMT (46). Suzuki et al. also demonstrated that miR-27b (a member of the miR-23/24/27 cluster), is a positive regulator of TGF- $\beta$-induced EndMT in mouse pancreatic microvascular endothelial cells (MS-1). The expression of miR-27b was upregulated by TGF- $\beta 1$ treatment and knockdown of miR-27 (by the locked nucleic acid miR-27b inhibitor) showed that suppression of TGF- $\beta$-induced EndMT. It was also found that miR-27 targets Elk1, neuropilin 2, Plexin A2 and Plexin D1 in the context of TGF- $\beta$-induced EndMT of MS-1 (47). Using miRNA array analysis, Li et al. found that miR-130a is upregulated in a monocrotaline pulmonary hypertension mouse model and the inhibition of miR-130a partially reversed TGF- $\beta$-induced EndMT in lung microvascular endothelial cells. It was also found that miR-130a is regulated by NF- $\mathrm{KB}$, and directly targets bone morphogenetic protein receptor 2 (48). Aside from TGF- $\beta$-induced EndMT and for hypoxia-induced EndMT, one group found that miR-126a-5p was elevated in hypoxiasubjected rat pulmonary microvascular endothelial cells (RPMECs), as well as a condition of persistent pulmonary hypertension in the newborn model. Concomitantly, decreased expression of PECAM- 1 and increased expression of $\alpha$-SMA in the hypoxic RPMECs was observed. Finally, it was found that inhibition of miR-126a-5p ameliorates hypoxia-induced EndMT (49). The EndMT-related miRNAs and target genes are summarized in Table 1 and Fig. 1.

\section{THE ENDOTHELIAL TO MESENCHYMAL TRANSITION AS A POTENTIAL THERAPEUTIC TARGET TO TREAT VARIOUS DISEASES}

Many studies have demonstrated that fibroblasts are implicated in a multitude of pathologies, and there is substantial evidence to indicate that they are the central mediator of pathological tissue remodeling (50). Several studies have demonstrated that a large proportion of fibroblasts found in damaged tissues are of endothelial origin via $\operatorname{EndMT}(7,51)$. These results suggest that the EndMT is an attractive prospective target with regard to prevention and treatment of many diseases. Indeed, the EndMT plays an essential role during development (52) and can also contribute to postnatal pathologies associated with many diseases such as fibrosis, neointima formation, diabetic complications, heterotopic ossification, Kawasaki disease and pulmonary arterial hypertension $(11,24,25,27,28,38$, 42-46, 48, 49, 53). Given that EndMT is closely involved in multiple diseases, the blocking of EndMT may represent a useful strategy for implementation in the treatment plans developed to combat human diseases. In addition, the miRNAs play a key role in the maintenance of homeostasis in the entire vasculature as they have the capacity to target multiple protein-encoding genes. Therefore, imbalances in the expression of miRNAs are closely related to the pathogenesis of many diseases via abnormal regulation of their target mRNAs. Thus, a strategy based on restoration of abnormal miRNA expression could have important therapeutic value for the treatment of various diseases. Finally, given the documented close relationship between EndMT and miRNAs as it occurs in various pathologies, modulation of miRNAs involved in EndMT processes could be a new therapeutic strategy for treatment of human diseases $(17,18)$. The therapeutic potential of modulation of miRNA expression in regulating the EndMT process in several diseases is summarized below.

As previously discussed, ECs represent a major source of the fibroblasts found in pathological fibrotic tissues via EndMT. In this context, let-7 miRNA plays a critical role in the regulation of EndMT via targeting components of TGF- $\beta$ signaling in HUVECs and HUAECs. It has also been shown that let-7 miRNA is regulated by FGF signaling $(11,24)$. It was demonstrated that let-7 miRNA and FGF receptor expression and phosphorylation were suppressed in diabetic condition(s). In addition, it was found that the endogenous antifibrotic peptide $\mathrm{N}$-acetyl-seryl-aspartyl-lysyl-proline (AcSDKP) restored let-7 miRNA levels by means of restoration of FGF receptor expression, as well as phosphorylation to normal levels. Thereby, AcSDKP inhibited EndMT, as evidenced by increased endothelial and reduced mesenchymal marker expression and, in turn, ameliorated diabetic kidney fibrosis. This suggests that AcSDKP could be a potential therapeutic option for diabetic kidney fibrosis as an endogenous antifibrotic molecule via inhibition of EndMT (24). The same group further showed the therapeutic potential of modulation of dysregulated miRNA during EndMT in the pathogenesis of diabetic kidney fibrosis (28). They found that DPP-4 could be induced in the diabetic kidney, and miR-29 expression was inhibited in diabetic mice (which identified DPP-4 as a direct target of miR-29). Thus, they identified the potential of DPP-4 inhibitor, which is generally used to treat diabetes mellitus type 2, for restoration of dysregulated miR-29 levels. As a result, DPP-4 inhibitor linagliptin restored miR-29 levels in diabetic kidney, and also found suppression of DPP-4 activity and protein expression. Thereby, EndMT was inhibited and, in turn, kidney fibrosis was ameliorated in diabetic kidney. These results suggest that linagliptin has potential therapeutic value for the restoration of normal kidney function in diabetic patients with kidney fibrosis via inhibition of EndMT (28).

Guo et al. revealed the role of kallistatin in the modulation of miRNA expression levels during TGF- $\beta$-induced-EndMT in 
HUVECs (54). Kallistatin is an endogenous protein and has an inhibitory effect on fibrosis via inhibition of TGF- $\beta$ expression (55). MiR-21 is a central activator of EndMT and fibrosis (45). They therefore attempted to find out whether kallistatin plays a key role in the association of EndMT and miR-21. It was shown that kallistatin suppressed TGF- $\beta$-induced EndMT and counteracted TGF- $\beta$-mediated miR-21 upregulation and activation of components of downstream pathways, such as Akt, NF- $\mathrm{kB}$ and matrix metalloproteinase 2. These results suggested that kallistatin plays a key role in protection against pathologic fibrotic diseases by suppressing miR-21 mediated activation of EndMT (54). Another recent study demonstrated the therapeutic potential of modulation of miR-483 by suppression of EndMT by means of the direct targeting of connective tissue growth factor (CTGF) in Kawasaki disease (53). They found that sera from Kawasaki disease patients led to reduction of miR-483 and induction of CTGF, as well as increased mesenchymal markers, such as $\alpha-S M A$, vimentin, and FSP-1 and decreased endothelial markers such as VE-cadherin and eNOS. They identified Krüppel-like factor 4 (KLF4) as the key transacting regulator that upregulates expression of miR-483 and, in turn, directly targets CTCF mRNA. In addition, it was found that atorvastatin activated the KLF4-miR-483 axis, inhibited CTGF expression, and alleviated EndMT using sera from Kawasaki disease patients. This suggests that statin could be a potential therapeutic option in Kawasaki disease, in part, through inhibition of the EndMT (53). Taken together, the current evidence indicates that the EndMT plays a key role in the pathogenesis of various diseases and that targeting of the EndMT is a novel therapeutic approach for many diseases. In addition, the restoration of dysregulated miRNA during EndMT to normal levels may be an attractive therapeutic option in the treatment of EndMT-related diseases. Thus, further studies are needed to clarify the underlying signaling mechanisms associated with EndMT and miRNA-based therapies to regulate EndMT are urgently needed.

\section{CONCLUSIONS AND FUTURE PERSPECTIVES}

MiRNAs play a key role in regulating EndMT by targeting multiple components associated with signaling pathways that regulate EndMT. As described above, many studies support that dysregulation of miRNAs in vascular ECs leads to activation of the EndMT process and contributes to the pathogenesis of human diseases. Thus, strategies that restore miRNA expression to physiological levels are attractive therapeutic opportunities for the treatment of human diseases via inhibition of EndMT. Although our current understanding of the molecular mechanisms underlying the miRNA-EndMT axis is advancing, more work is still required to better understand the complex network of miRNAs and their target mRNAs involved in the EndMT process. In addition, understanding of the regulation of the reversible biological process of EndMT is necessary for the prevention and treatment of many diseases. In conclusion, studies of miRNA involved in EndMT will provide new insights into the molecular mechanisms of a broad variety of human pathologies and the identification of potential targets that are able to inhibit EndMT, will provide effective therapeutic drugs for the treatment of human diseases.

\section{ACKNOWLEDGEMENTS}

This research was supported by the Basic Science Research Program through the National Research Foundation of Korea (NRF) funded by the minister of Education, Science and Technology (NRF-2016R1A5A1011974 and NRF-2016R1C1B 2006591 to J.K).

\section{CONFLICTS OF INTEREST}

The authors have no conflicting interests.

\section{REFERENCES}

1. Michiels C (2003) Endothelial cell functions. J Cell Physiol 196, 430-443

2. Markwald RR, Fitzharris TP and Smith WN (1975) Sturctural analysis of endocardial cytodifferentiation. Dev Biol 42, 160-180

3. Armstrong EJ and Bischoff J (2004) Heart valve development: endothelial cell signaling and differentiation. Circ Res 95, 459-470

4. Nakajima $Y$, Yamagishi T, Hokari $\mathrm{S}$ and Nakamura $\mathrm{H}$ (2000) Mechanisms involved in valvuloseptal endocardial cushion formation in early cardiogenesis: roles of transforming growth factor (TGF)-beta and bone morphogenetic protein (BMP). Anat Rec 258, 119-127

5. van Meeteren LA and ten Dijke P (2012) Regulation of endothelial cell plasticity by TGF-beta. Cell Tissue Res 347, 177-186

6. Krenning G, Zeisberg EM and Kalluri R (2010) The origin of fibroblasts and mechanism of cardiac fibrosis. J Cell Physiol 225, 631-637

7. Zeisberg EM, Potenta S, Xie L, Zeisberg M and Kalluri R (2007) Discovery of endothelial to mesenchymal transition as a source for carcinoma-associated fibroblasts. Cancer Res 67, 10123-10128

8. Ghosh AK, Quaggin SE and Vaughan DE (2013) Molecular basis of organ fibrosis: potential therapeutic approaches. Exp Biol Med (Maywood) 238, 461-481

9. Arciniegas E, Frid MG, Douglas IS and Stenmark KR (2007) Perspectives on endothelial-to-mesenchymal transition: potential contribution to vascular remodeling in chronic pulmonary hypertension. Am J Physiol Lung Cell Mol Physiol 293, L1-L8

10. Cooley BC, Nevado J, Mellad J et al (2014) TGF-beta signaling mediates endothelial-to-mesenchymal transition (EndMT) during vein graft remodeling. Sci Transl Med 6, $227 \mathrm{ra3} 4$ 
11. Chen PY, Qin L, Barnes C et al (2012) FGF regulates TGF-beta signaling and endothelial-to-mesenchymal transition via control of let-7 miRNA expression. Cell Rep 2, 1684-1696

12. Yao $Y$, Jumabay $M$, Ly A, Radparvar M, Cubberly MR and Bostrom KI (2013) A role for the endothelium in vascular calcification. Circ Res 113, 495-504

13. Chen PY, Qin L, Baeyens N et al (2015) Endothelial-tomesenchymal transition drives atherosclerosis progression. J Clin Invest 125, 4514-4528

14. Maddaluno L, Rudini N, Cuttano R et al (2013) EndMT contributes to the onset and progression of cerebral cavernous malformations. Nature 498, 492-496

15. Potenta S, Zeisberg E and Kalluri R (2008) The role of endothelial-to-mesenchymal transition in cancer progression. Br J Cancer 99, 1375-1379

16. Kovacic JC, Mercader $\mathrm{N}$, Torres $\mathrm{M}$, Boehm $\mathrm{M}$ and Fuster $\checkmark$ (2012) Epithelial-to-mesenchymal and endothelial-tomesenchymal transition: from cardiovascular development to disease. Circulation 125, 1795-1808

17. Lee A, McLean D, Choi J, Kang H, Chang W and Kim J (2014) Therapeutic implications of microRNAs in pulmonary arterial hypertension. BMB Rep 47, 311-317

18. Kim JD, Lee A, Choi J et al (2015) Epigenetic modulation as a therapeutic approach for pulmonary arterial hypertension. Exp Mol Med 47, e175

19. Hobert $O$ (2008) Gene regulation by transcription factors and microRNAs. Science 319, 1785-1786

20. Pardali E, Sanchez-Duffhues G, Gomez-Puerto MC and Ten Dijke P (2017) TGF-beta-Induced EndothelialMesenchymal Transition in Fibrotic Diseases. Int J Mol Sci 18

21. Noseda M, McLean G, Niessen K et al (2004) Notch activation results in phenotypic and functional changes consistent with endothelial-to-mesenchymal transformation. Circ Res 94, 910-917

22. Perez L, Munoz-Durango N, Riedel CA et al (2017) Endothelial-to-mesenchymal transition: Cytokine-mediated pathways that determine endothelial fibrosis under inflammatory conditions. Cytokine Growth Factor Rev 33, 41-54

23. Welch-Reardon KM, Wu N and Hughes CC (2015) A role for partial endothelial-mesenchymal transitions in angiogenesis? Arterioscler Thromb Vasc Biol 35, 303-308

24. Nagai T, Kanasaki M, Srivastava SP et al (2014) N-acetylseryl-aspartyl-lysyl-proline inhibits diabetes-associated kidney fibrosis and endothelial-mesenchymal transition. Biomed Res Int 2014, 696475

25. Zhang H, Hu J and Liu L (2017) MiR-200a modulates TGF-beta1-induced endothelial-to-mesenchymal shift via suppression of GRB2 in HAECs. Biomed Pharmacother 95, 215-222

26. Correia AC, Moonen IR, Brinker MG and Krenning $G$ (2016) FGF2 inhibits endothelial-mesenchymal transition through microRNA-20a-mediated repression of canonical TGF-beta signaling. J Cell Sci 129, 569-579

27. Sun Y, Cai J, Yu S, Chen S, Li F and Fan C (2016) MiR-630 Inhibits Endothelial-Mesenchymal Transition by Targeting Slug in Traumatic Heterotopic Ossification. Sci Rep 6, 22729
28. Kanasaki K, Shi S, Kanasaki M et al (2014) Linagliptinmediated DPP-4 inhibition ameliorates kidney fibrosis in streptozotocin-induced diabetic mice by inhibiting endothelial-to-mesenchymal transition in a therapeutic regimen. Diabetes 63, 2120-2131

29. Korpal M, Lee ES, Hu G and Kang Y (2008) The miR-200 family inhibits epithelial-mesenchymal transition and cancer cell migration by direct targeting of E-cadherin transcriptional repressors ZEB1 and ZEB2. J Biol Chem 283, 14910-14914

30. Zhang S, Weinheimer C, Courtois M et al (2003) The role of the Grb2-p38 MAPK signaling pathway in cardiac hypertrophy and fibrosis. J Clin Invest 111, 833-841

31. Ge S, Xie J, Liu F, He J and He J (2015) MicroRNA-19b reduces hepatic stellate cell proliferation by targeting GRB2 in hepatic fibrosis models in vivo and in vitro as part of the inhibitory effect of estradiol. J Cell Biochem $116,2455-2464$

32. Fafeur V, Terman BI, Blum J and Bohlen P (1990) Basic FGF treatment of endothelial cells down-regulates the 85-KDa TGF beta receptor subtype and decreases the growth inhibitory response to TGF-beta 1 . Growth Factors 3, 237-245

33. Papetti M, Shujath J, Riley KN and Herman IM (2003) FGF-2 antagonizes the TGF-beta1-mediated induction of pericyte alpha-smooth muscle actin expression: a role for myf-5 and Smad-mediated signaling pathways. Invest Ophthalmol Vis Sci 44, 4994-5005

34. Ramos C, Becerril C, Montano M et al (2010) FGF-1 reverts epithelial-mesenchymal transition induced by TGF-\{beta\}1 through MAPK/ERK kinase pathway. Am J Physiol Lung Cell Mol Physiol 299, L222-231

35. Lee JG, Ko MK and Kay EP (2012) Endothelial mesenchymal transformation mediated by IL-1beta-induced FGF-2 in corneal endothelial cells. Exp Eye Res 95, 35-39

36. Lee HT, Lee JG, Na M and Kay EP (2004) FGF-2 induced by interleukin- 1 beta through the action of phosphatidylinositol 3-kinase mediates endothelial mesenchymal transformation in corneal endothelial cells. J Biol Chem $279,32325-32332$

37. Aird WC (2007) Phenotypic heterogeneity of the endothelium: I. Structure, function, and mechanisms. Circ Res 100, 158-173

38. Lagendijk AK, Goumans MJ, Burkhard SB et al (2011) MicroRNA-23 restricts cardiac valve formation by inhibiting Has2 and extracellular hyaluronic acid production. Circ Res 109, 649-657

39. Bayoumi AS, Teoh JP, Aonuma T et al (2017) MicroRNA-532 protects the heart in acute myocardial infarction, and represses prss23, a positive regulator of endothelial-to-mesenchymal transition. Cardiovasc Res 113, 1603-1614

40. Bijkerk R, de Bruin RG, van Solingen C et al (2012) MicroRNA-155 functions as a negative regulator of RhoA signaling in TGF-beta-induced endothelial to mesenchymal transition. Microrna 1, 2-10

41. Ruderman NB, Williamson JR and Brownlee M (1992) Glucose and diabetic vascular disease. FASEB J 6, 2905-2914

42. Cao Y, Feng B, Chen S, Chu Y and Chakrabarti S (2014) 
Mechanisms of endothelial to mesenchymal transition in the retina in diabetes. Invest Ophthalmol Vis Sci 55, 7321-7331

43. Feng B, Cao $Y$, Chen $S$, Chu $X, C h u ~ Y$ and Chakrabarti S (2016) miR-200b Mediates Endothelial-to-Mesenchymal Transition in Diabetic Cardiomyopathy. Diabetes 65, 768-779

44. Geng HZ and Guan J (2017) MiR-18a-5p inhibits endothelial mesenchymal transition and cardiac fibrosis through the Notch2 pathway. Biochem Biophys Res Commun 491, 329-336

45. Kumarswamy R, Volkmann I, Jazbutyte V, Dangwal S, Park DH and Thum T (2012) Transforming Growth Factor-beta-Induced Endothelial-to-Mesenchymal Transition Is Partly Mediated by MicroRNA-21. Arterioscler Thromb Vasc Biol 32, 361-369

46. Ghosh AK, Nagpal V, Covington JW, Michaels MA and Vaughan DE (2012) Molecular basis of cardiac endothelial-to-mesenchymal transition (EndMT): differential expression of microRNAs during EndMT. Cell Signal 24, 1031-1036

47. Suzuki HI, Katsura A, Mihira H, Horie M, Saito A and Miyazono K (2017) Regulation of TGF-beta-mediated endothelial-mesenchymal transition by microRNA-27. J Biochem 161, 417-420

48. Li L, Kim IK, Chiasson V, Chatterjee P and Gupta S (2017) NF-kappaB mediated miR-130a modulation in lung microvascular cell remodeling: Implication in pulmonary hypertension. Exp Cell Res 359, 235-242

49. Xu YP, He Q, Shen Z et al (2017) MiR-126a-5p is involved in the hypoxia-induced endothelial-to-mesenchymal transition of neonatal pulmonary hypertension. Hypertens Res $40,552-561$

50. Kendall RT and Feghali-Bostwick CA (2014) Fibroblasts in fibrosis: novel roles and mediators. Front Pharmacol 5, 123

51. Zeisberg EM, Tarnavski O, Zeisberg $M$ et al (2007) Endothelial-to-mesenchymal transition contributes to cardiac fibrosis. Nat Med 13, 952-961

52. Lin F, Wang N and Zhang TC (2012) The role of endothelial-mesenchymal transition in development and pathological process. IUBMB Life 64, 717-723

53. He M, Chen Z, Martin M et al (2017) miR-483 Targeting of CTGF Suppresses Endothelial-to-Mesenchymal Transition: Therapeutic Implications in Kawasaki Disease. Circ Res 120, 354-365

54. Guo Y, Li P, Bledsoe G, Yang ZR, Chao L and Chao J (2015) Kallistatin inhibits TGF-beta-induced endothelialmesenchymal transition by differential regulation of microRNA-21 and eNOS expression. Exp Cell Res 337, 103-110

55. Shen B, Hagiwara M, Yao YY, Chao L and Chao J (2008) Salutary effect of kallistatin in salt-induced renal injury, inflammation, and fibrosis via antioxidative stress. Hypertension 51, 1358-1365 